\title{
Novel Approach Based on Genetic Algorithm for Adaptive Resource Management in Software-Defined Networks
}

\author{
Mohammed Najm Abdullah ${ }^{1}$, Afrah Salman Dawood ${ }^{1}$ \\ Department of Computer Engineering, University of Technology, Baghdad, Iraq ${ }^{1}$
}

\begin{abstract}
Resource management in Software-Defined Networking (SDN) is an important aspect in this brand new approach of networking. Implementing an efficient resource management with high Quality-of-Service (QoS) requirements like low delay or high bandwidth is a big challenge. Dynamic and adaptive solutions can be benefit for improving the performance of these networks. In this paper, we propose a novel approach of using genetic algorithm with SDN architecture and FNSS toolchain for better optimization of adaptive resource management with testbeds on datacenter topologies with video streams run on VLC media player to evaluate the performance of our solution. We explained the testbeds on the test video (delay and bandwidth) by implementing the adaptive solution using Genetic Algorithm (GA) for adaptively selecting the capacity according to link delay which then reduces the overall timeof packet delivery and increases the bandwidth. The final results shows an improvementin reducing the average delay values of $94.4 \%$ when using OVS controller and $90.1 \%$ when using Floodlight controller, and an improvement in bandwidth of 950 Mbps- 1.82 Mbps to 37.075 Mbps- 42.9 Mbps in OVS controller and 952 Mbps- 1.7 Mbps to 22.97 Mbps- 27.45 Mbps in Floodlight controller.
\end{abstract}

Keywords: SDN, Adaptive Resource Management, Fast Network Simulation Setup (FNSS), OVS Controller, Floodlight Controller, Mininet, Genetic Algorithm, Datacenter topology.

\section{INTRODUCTION}

Software-Defined Networking is the new concept of and tools used in this work. In Section IV, we presents our networking that separates the control plane from the data proposal, and finally, Sections V and VI describes results plane [1] to simplify network management and improve and performance evaluation and conclusions, respectively. data transmission and overcome difficulties of traditional networks. The environment of these networks, known as Software Defined Environment (SDE), is responsible for optimizing Resource Management (RM) in network computing infrastructure [2]. SDN provides a great management, scalability, performance and other features [3]. Currently, resource management is based on parameters like link capacity, delay, bandwidth, etc.

Algorithms can be analyzed in different methods such as theaverage-case analysis for measuring the average performance on problems of size n. Adaptive analysis basically contrasts the performance of the algorithm to the difficulty of the related issue [9]. Genetic algorithm is one of the best adaptive algorithms which is a heuristic random search mechanism (i.e. evolutionary process) [10].These adaptive algorithms can be used in traditional networks and Software-Defined Networks for better resource utilization. The possibilities of using such adaptive algorithms in such modular networks has already been researched and discussed in [11].

In this paper, we propose a novel approach of optimizing resources in these networks based on adaptive algorithms (i.e. genetic algorithm) to enhance QoS requirements. The rest of the paper is arranged as follows: related work is described in Section II, Section III explains basic concepts

\section{RELATED WORK}

Since resource management is an important concept in any system, SDN researchers are moving towards optimizing and improving these resources. The new orientation is to adaptively optimize RM using adaptive algorithms in order to increase performance and maintenance of SND. Dissertation in reference [3] focused on resources in the SND structure and proposed an EPM application which is a novel SDN-based structure for the purpose of assigning privacy preserving multicast in cloud data center.

This dissertation then optimized limited resources to guarantee the security in SDN and presented the design of an investigative approach induced by rule placement in the facet of traffic changes and mobile devices movement. Reference [4], managed Virtual Network's (VN) resources efficiently by using SDN's control plane and extending SDN controller to monitor resource utilization in VNs. Researcher in this reference showed an improvement in VN's acceptance ratio by around $40 \%$ and a reduction of VN's resource costs by around $10 \%$. The paper in reference [5], presented a new arrangement of SDN-based management and control for fixed basis network that tools up support for both static and dynamic resource 
management applications. The authors in this reference developed an employment algorithm for determining the allocation of managers and controllers in the proposed distributed management and control layer, after that, they selected two specific applications with adaptive loadbalancing and energy management objective and explained how the proposed layer be content with the selected applications. Reference [6], explained a summarization on techniques used for resource management with available SDN tactics based on OpenFlow protocol. Authors in [7] presented a theoretical suggestion to distribute and balance the load on controllers based on genetic algorithm when imbalance is detected.The doctoral thesis in [8]proposed and implemented an architecture of cloud platform provided with a mechanism that impacts the dynamic nature of SDN and a use of a virtualization-based technique to protect virtual appliances.

In summary, we can see that our proposal is a novel in this field by using adaptive algorithms (i.e. genetic algorithm) for optimizing resources in SDN using a combination of network software like Mininet, FNSS, OVS controller, Floodlight controller, and VLC media player and different packages like PULP for solving ILP problems.

\section{BASIC CONCEPTS}

This section describes basic terms and concepts used in this work such as Mininet, OVS and Floodlight Controller and FNSS toolchain.Mininetis a software emulator used to run SDN topologies with a suitable controller [12]. Emulator is hardware or software that enables one computer system (i.e. host) to behave like another computer system (i.e. guest).

Two types of controllers have been used in this paper which are OVS Controller [13] (i.e. Open Virtual Switch Controller) that is a multilayer virtual switch authorized under the open source Apache 2.0 permit. It can operate both as a software-based network switch running within the virtual machine (VM) hypervisors, and as the control stack for dedicated switching hardware; as a result, it has been ported to multiple virtualization platforms, switching chipsets, and networking hardware accelerators. The other controller is the Floodlight Project Controller [14] that is introduced by Big Switch Networks and is based on OpenFlow protocol. This controller can be invaluable or precious to SDN researchers because of its sensible REST API and the use of Java programming language; besides to the satisfying results it offers.

A helpful toolchain named FNSS [1] (Fast Network Simulation Setup) has also been used, which is a set of programming tools used for implementing convoluted software development function or to create a software product. FNSS is based on a set of scenarios in its work as shown in Figure 1. The completed scenario has to be released to FNSS to be achieved in the prioritized goaled software simulator or emulator.

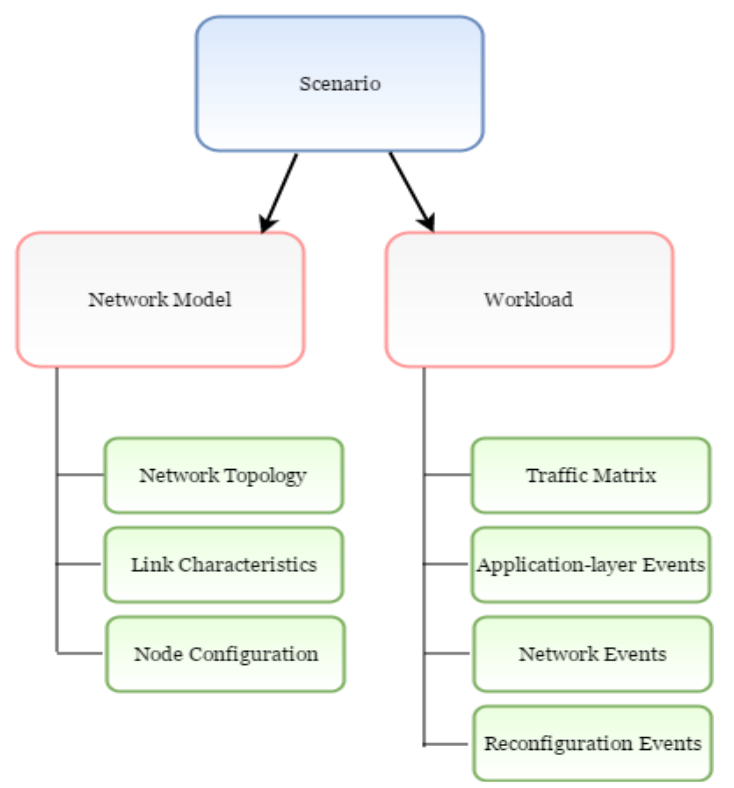

Figure 1: The Components of a Complete FNSS Scenario

\section{PROPOSED ADAPTIVE SOLUTION USING GENETIC ALGORITHM}

Resource management optimization has been implemented in this paper based on using adaptive solution by genetic algorithm to find the best capacity for each link in the network according to its current delay, and then assigns a suitable capacity to increase the bandwidth and reduce the delay according to new link capacity. Genetic algorithm has been implemented using regular phases as shown in algorithm 1. The proposed solution has been implemented in a python programming language according to algorithm2.

Algorithm1: Basic steps of genetic algorithm

Step 1. Generate the required initial population

Step 2. Find the grade of the population

Step 3. For specific range; repeat

a. Prepare parents

b. Randomly add other individuals to promote genetic diversity

c. Mutate some individuals

d. Crossover parents to create children

e. Make children the new population

Step 4. Return the best capacity

Algorithm2: The steps of the proposed solution

Step 1. Import required libraries

Step 2. Set the desired datacenter topology with links and cores

Step 3. Set the delay to 'ms'

Step 4. Assign constant weights for each link in topology

Step 5. Set adaptive capacities to each link according to algorithm1 with 'Mbps' as a capacity units and 'bytes' as a buffer units

Step 6. Set buffer sizes according to bandwidth and delay product

Step 7. Draw the topology with the help of ' $\mathrm{nx}$ ' library 
Step 8. Set video transferring properties Step 9. Convert FNSS topology to Mininet Step 10. Launch the new Mininet topology with the required controller and start the network

Step 11. Perform video transferring with VLC media player and see the results

Step 12. Stop the network and implement clean command to clear all Ethernet links to be able to run new networks.

\section{RESULTS}

The proposed solution has been implemented and tested using Mininet emulator, OVS controller, python package, networkx library, PULP library, and FNSS toolchain. All these software tools have been implemented on Ubuntu 16.04 operating system. We implement our proposed solution on three types of network topologies and record the results according; these topologies are Two Tier Datacenter Topology, Three Tier Datacenter Topology, and Fat Tree Datacenter Topology for different number to cores and levels (see figures 2 through 5). We record the delay and bandwidth values according to three types of packets (1 Kbyte, 24 Kbyte, and 64.4 Kbyte) through the ping command between different hosts and results are shown in tables 1 through 6.
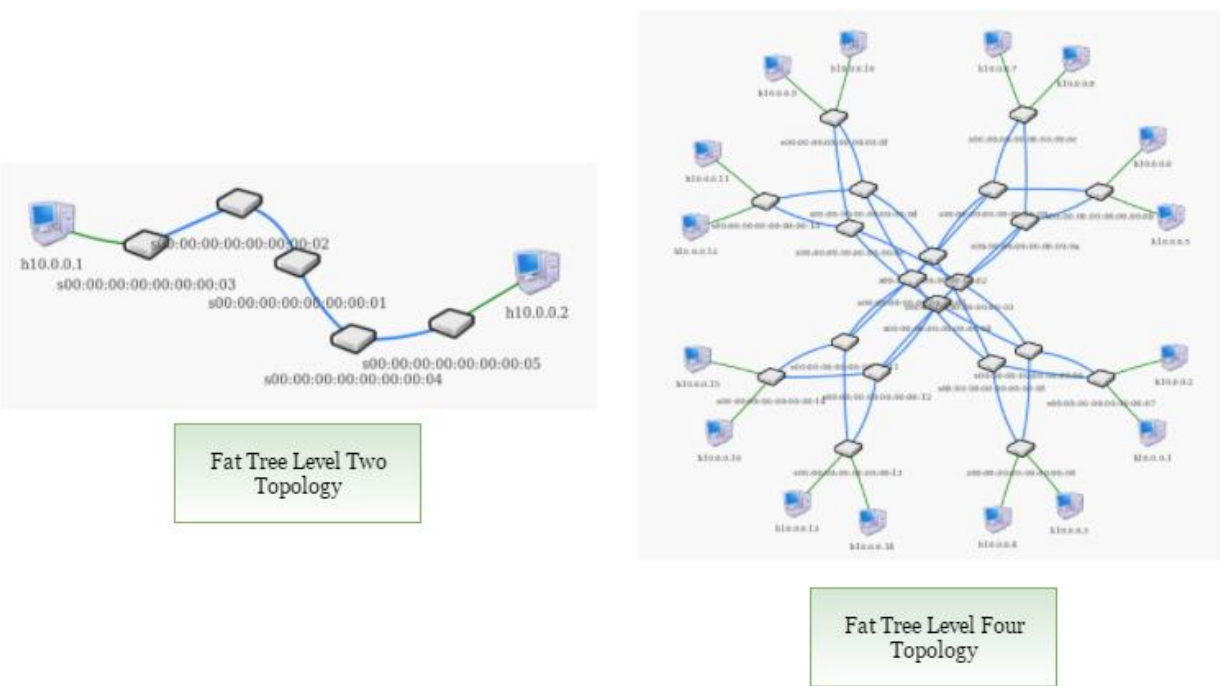

Figure2: Fat Tree Datacenter Topology

Table 1: Floodlight Simulation Results of Fat Tree Datacenter Topology

\begin{tabular}{|c|c|c|}
\hline \multirow{2}{*}{$\begin{array}{c}\text { Packet } \\
\text { Size }\end{array}$} & Before Using The Proposed Solution & $\begin{array}{c}\text { After Using The Adaptive Solution with Genetic } \\
\text { Algorithm }\end{array}$ \\
\cline { 2 - 3 } 1 Kbyte & 17.544 & 20.466 \\
\hline 24 Kbyte & 389.022 & 34.927 \\
\hline 64.4 Kbyte & 1047.974 & 167.248 \\
\hline
\end{tabular}

Table 2: OVS Simulation Results of Fat Tree Datacenter Topology

\begin{tabular}{|c|c|c|}
\hline \multirow{2}{*}{$\begin{array}{c}\text { Packet } \\
\text { Size }\end{array}$} & Before Using The Proposed Solution & After Using The Adaptive Solution with Genetic Algorithm \\
\hline & 12.962 & 13.339 \\
\hline 1 Kbyte & 375.67 & 22.191 \\
\hline 24 Kbyte & 1029.848 & 39.210 \\
\hline
\end{tabular}

Table 3: OVS Simulation Results of Two Tier Datacenter Topology

\begin{tabular}{|c|c|c|}
\hline \multirow{2}{*}{ Packet Size } & \multicolumn{2}{|c|}{ Average Delay in (ms) } \\
\cline { 2 - 3 } & Before Using The Proposed Solution & $\begin{array}{c}\text { After Using The Adaptive Solution with Genetic } \\
\text { Algorithm }\end{array}$ \\
\hline 1 Kbyte & 8.831 & 16.139 \\
\hline 24 Kbyte & 371.503 & 33.729 \\
\hline 64.4 Kbyte & 1025.229 & 50.644 \\
\hline
\end{tabular}




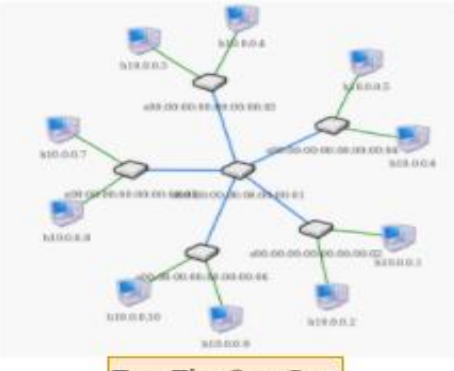

Two Tier One Core Topology

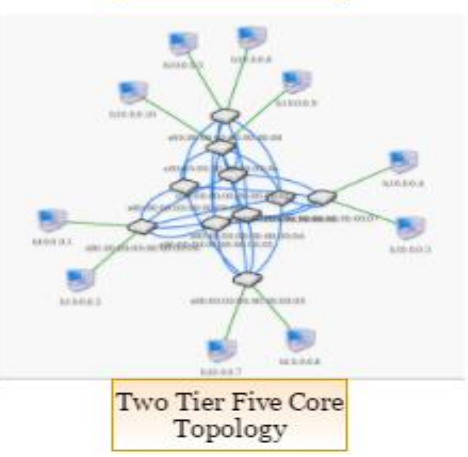

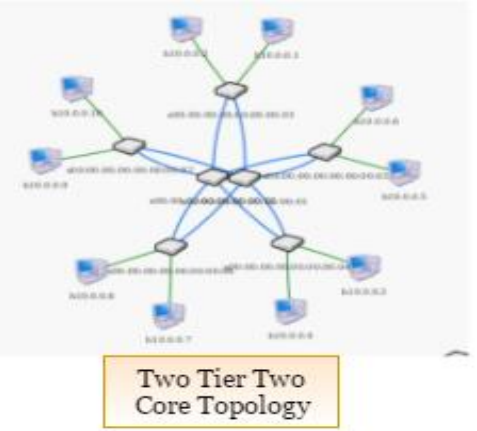

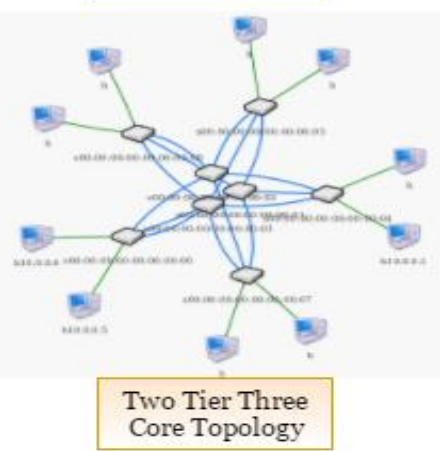

Figure3: Two Tier Datacenter Topology

Table 4: Floodlight Simulation Results of Two Tier Datacenter Topology

\begin{tabular}{|c|c|c|}
\hline \multirow{2}{*}{$\begin{array}{c}\text { Packet } \\
\text { Size }\end{array}$} & \multicolumn{2}{|c|}{ Average Delay in (ms) } \\
\cline { 2 - 3 } 1 Kbyte & 19.721 & After Using The Adaptive Solution with Genetic Algorithm \\
\hline 24 Kbyte & 370.901 & 43.7644 \\
\hline 64.4 Kbyte & 1038.980 & 43.1662 \\
\hline
\end{tabular}
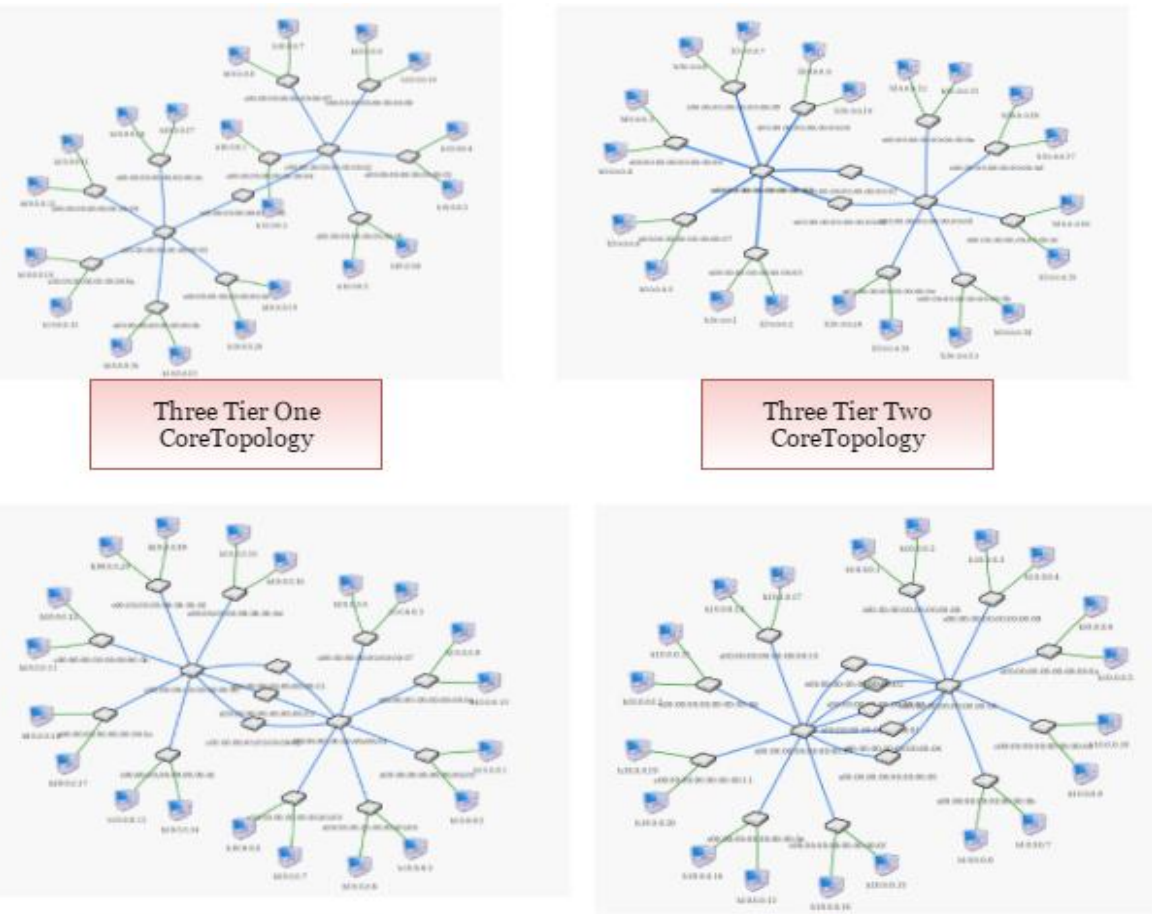

Three Tier Three CoreTopology

Three Tier Five CoreTopology

Figure4: Three Tier Datacenter Topology 
Vol. 6, Issue 1, January 2017

Table 5: OVS Simulation Results of Three Tier Datacenter Topology

\begin{tabular}{|c|c|c|}
\hline \multirow{2}{*}{$\begin{array}{c}\text { Packet } \\
\text { Size }\end{array}$} & Before Using The Proposed Solution & $\begin{array}{c}\text { After Using The Adaptive Solution with Genetic } \\
\text { Algorithm }\end{array}$ \\
\cline { 2 - 3 } 1 Kbyte & 17.712 & 8.859 \\
\hline 24 Kbyte & 385.549 & 18.1145 \\
\hline 64.4 Kbyte & 1037.191 & 34.8235 \\
\hline
\end{tabular}

Table 6: Floodlight Simulation Results of Three Tier Datacenter Topology

\begin{tabular}{|c|c|c|}
\hline \multirow{2}{*}{ Packet Size } & \multicolumn{2}{|c|}{ Average Delay in (ms) } \\
\cline { 2 - 3 } & Before Using The Proposed Solution & $\begin{array}{c}\text { After Using The Adaptive Solution with Genetic } \\
\text { Algorithm }\end{array}$ \\
\hline 1 Kbyte & 23.689 & 18.0775 \\
\hline 24 Kbyte & 388.254 & 27.95375 \\
\hline 64.4 Kbyte & 1044.579 & 57.91575 \\
\hline
\end{tabular}

\section{PERFORMANCE EVALUATION AND CONCLUSION}

By comparing the results from the previous section, we can see the efficiency of our algorithm. The performance of OVS controller can be shown in figures 5, 6, and 7; As a comparison in performance between OVS and while the performance of Floodlight controller can be Floodlight controllers, we can see that OVS controller shown in figures 8,9 , and 10 . The OVS controller reduced performs better than Floodlight controller in fat tree the delay by $94.4 \%$ while the bandwidth increased from topologies whereas Floodlight controller performs better 950 Mbps- 1.82 Mbps to 37.075 Mbps- 42.9 Mbps as an than OVS in other types of datacenter topologies.It is average. The Floodlight controller reduced the delay by worth mentioning that although OVS performs better than 90.1\% while the bandwidth increased from 952 Mbps- 1.7 Floodlight controller in the percentage but OVS controller Mbps to22.97 Mbps- 27.45 Mbps as an average. We can cannot maintain high number of cores in datacenter see the performance of our proposed solution by running topology while Floodlight controller can work properly videos of 2.00 MByte and 5.00 Mbyte in VLC media with more than one core in the same topology.

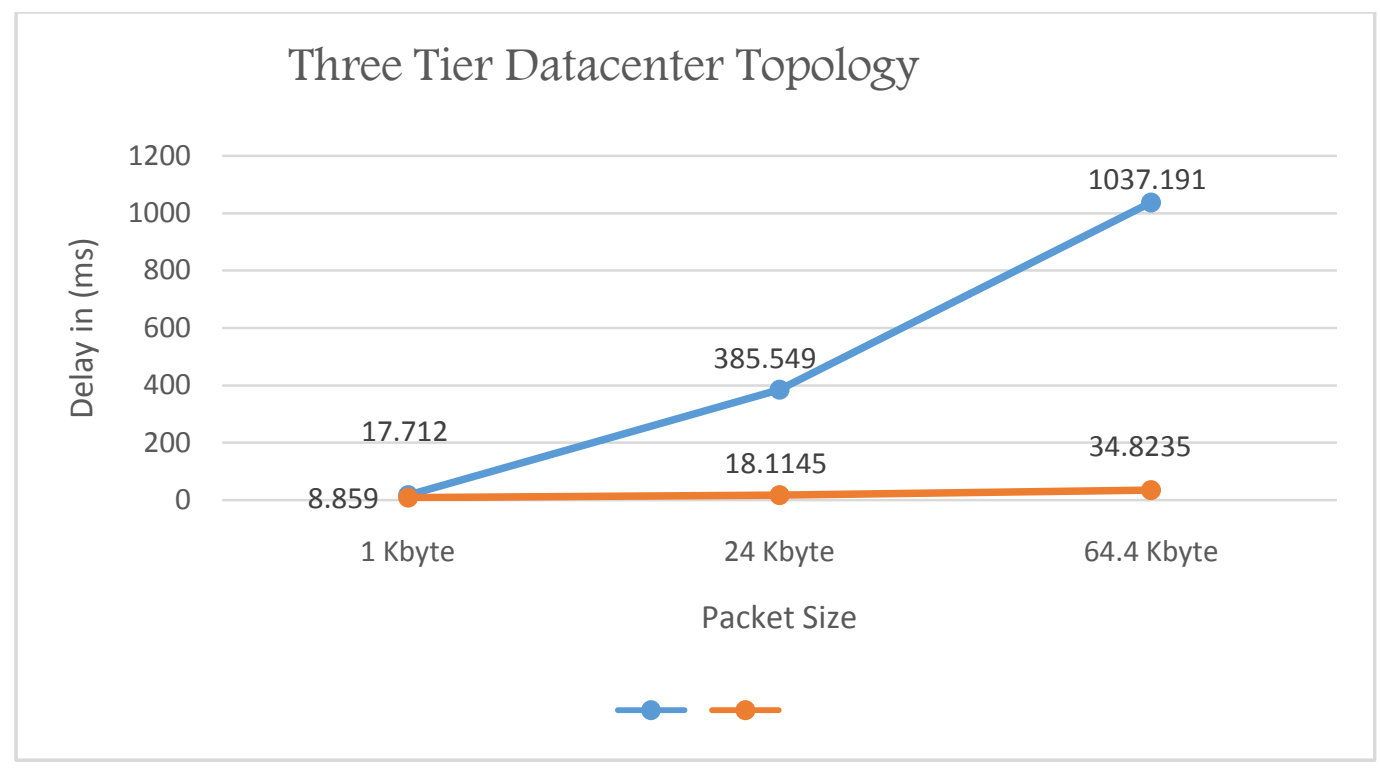

Figure5: Performance Evaluation for Three TierDatacenter Topology using OVS Controller 
Vol. 6, Issue 1, January 2017

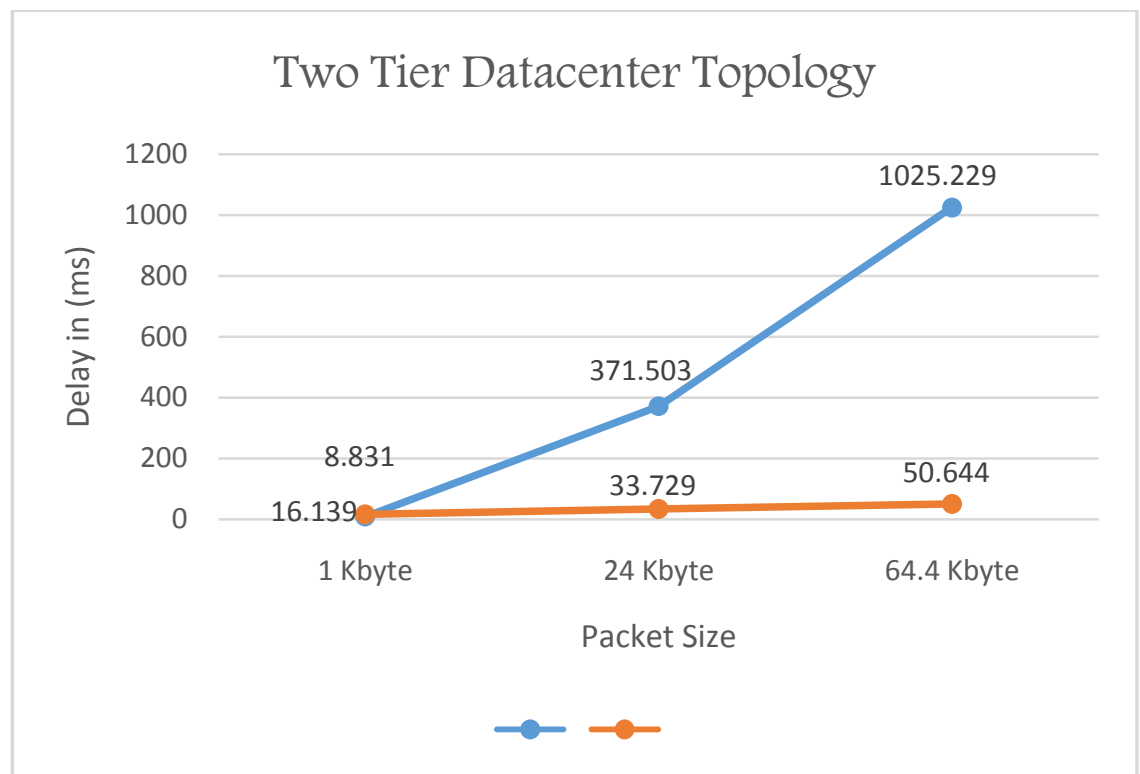

Figure6: Performance Evaluation for Two Tier Datacenter Topology using OVS Controller

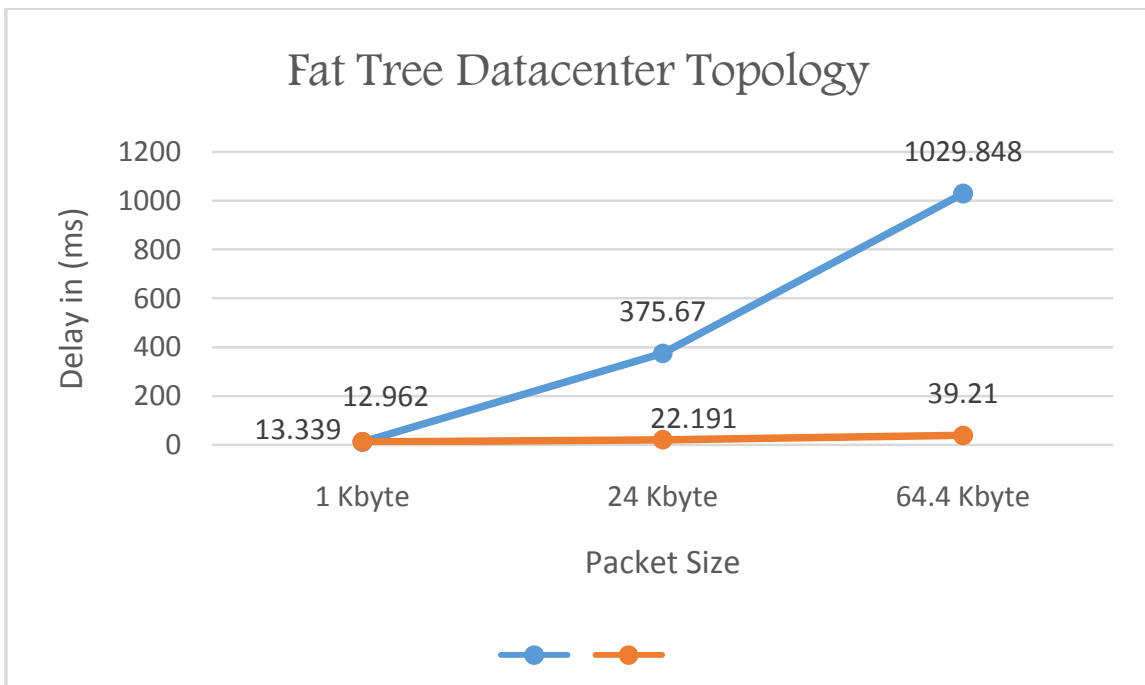

Figure7: Performance Evaluation for Fat Tree Datacenter Topology using OVS Controller

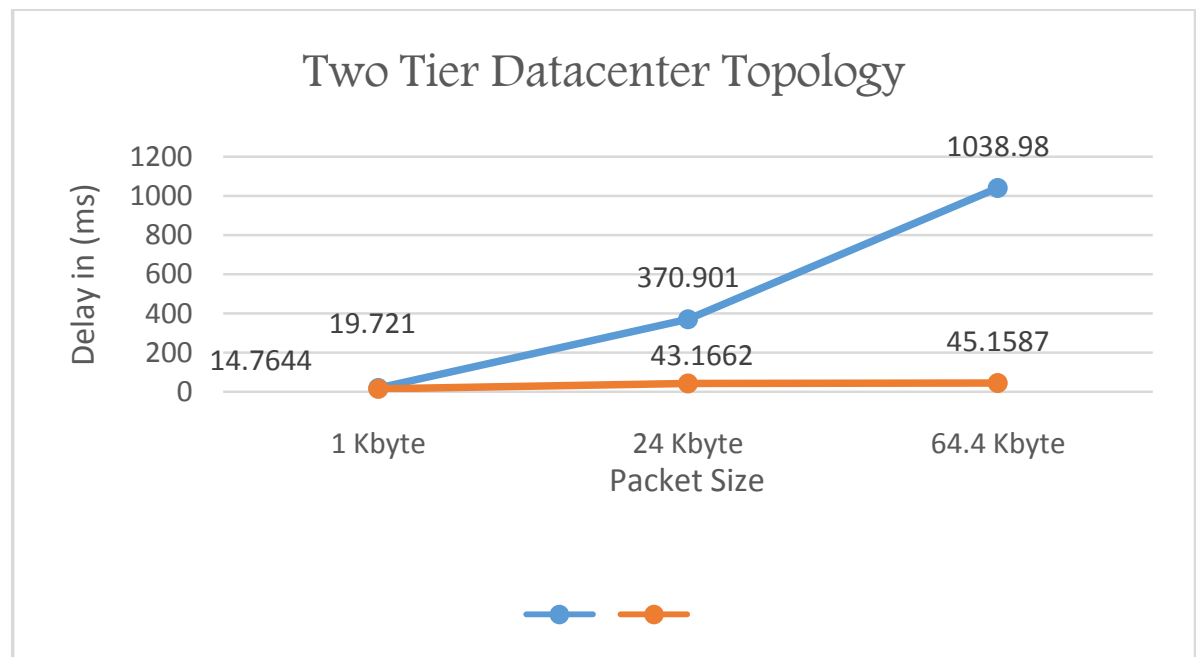

Figure8: Performance Evaluation for Two Tier Datacenter Topology using Floodlight Controller 
International Journal of Advanced Research in Computer and Communication Engineering ISO 3297:2007 Certified

Vol. 6, Issue 1, January 2017

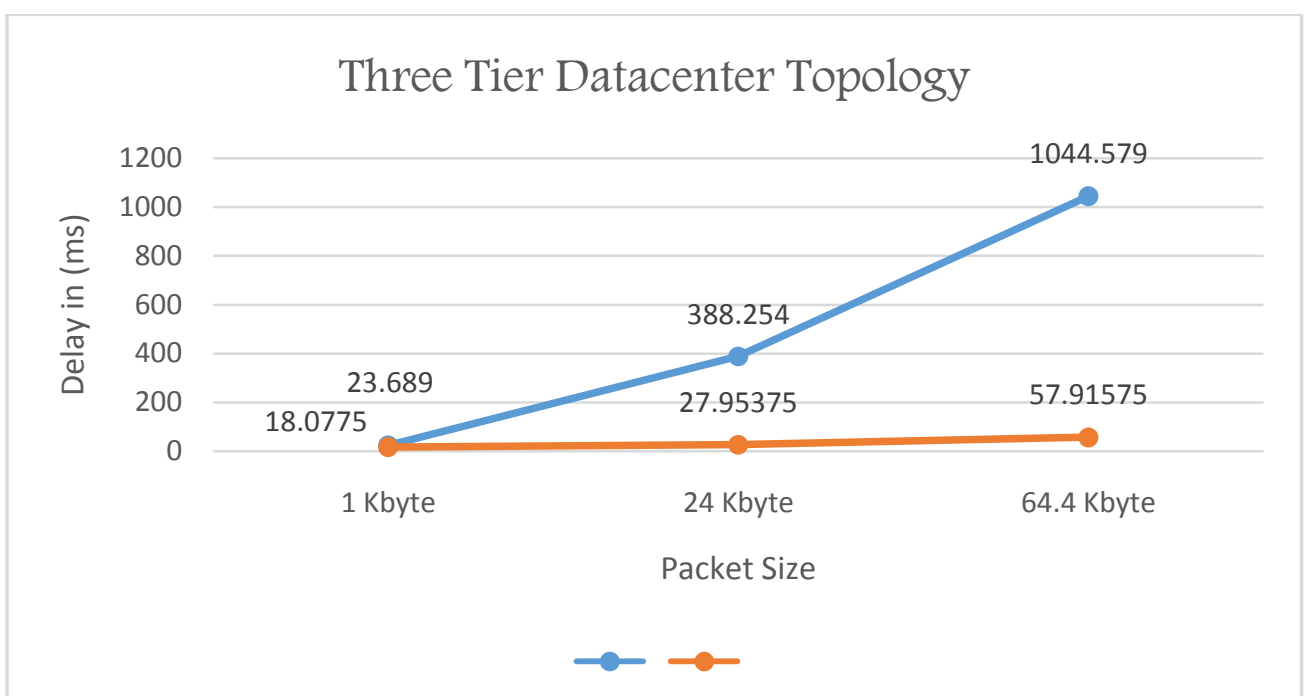

Figure9: Performance Evaluation for Three Tier Datacenter Topology using Floodlight Controller

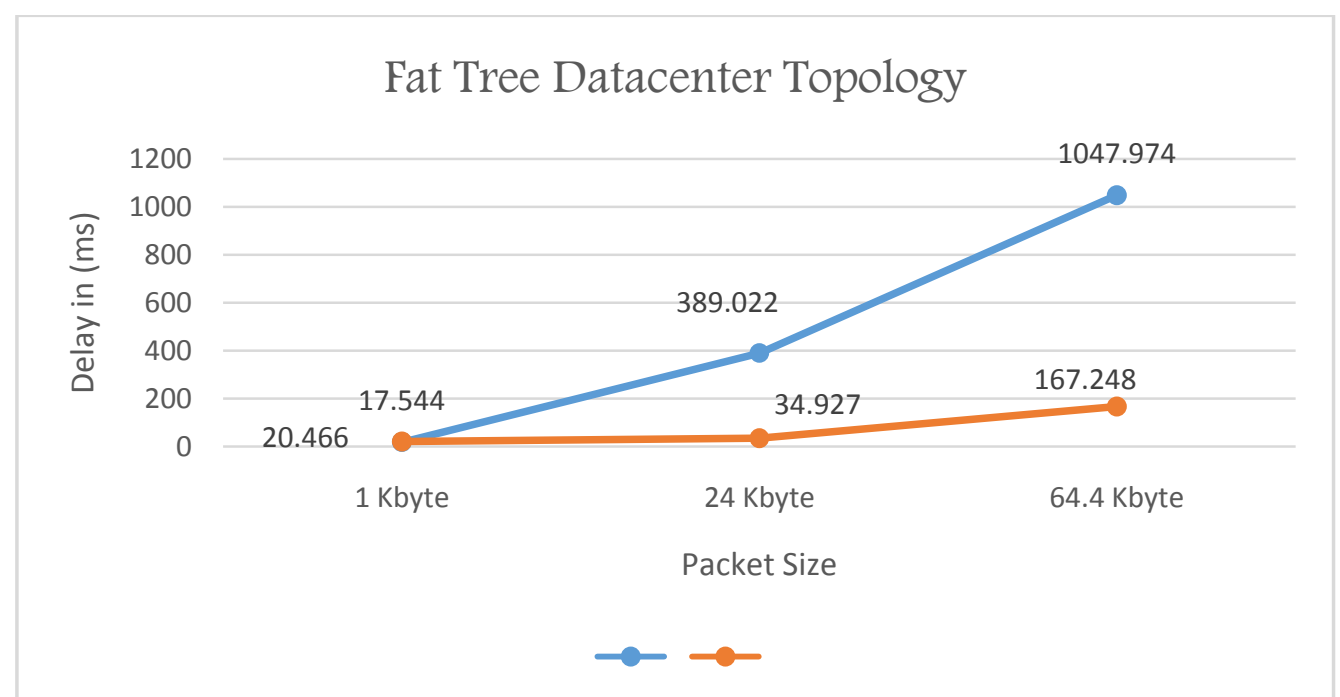

Figure10: Performance Evaluation for Fat Tree Datacenter Topology using Floodlight Controller

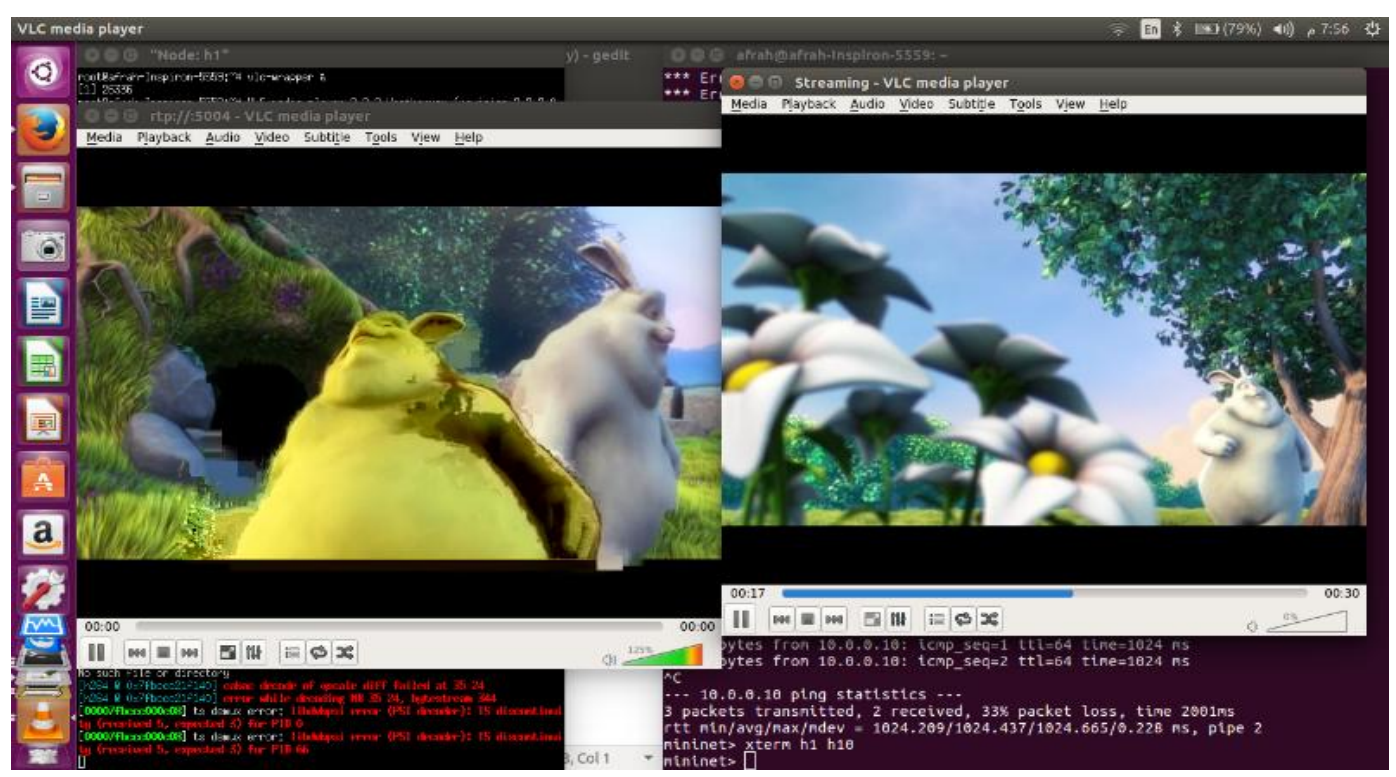

Figure11: Video Performance before Implementing Genetic Algorithm 
Vol. 6, Issue 1, January 2017

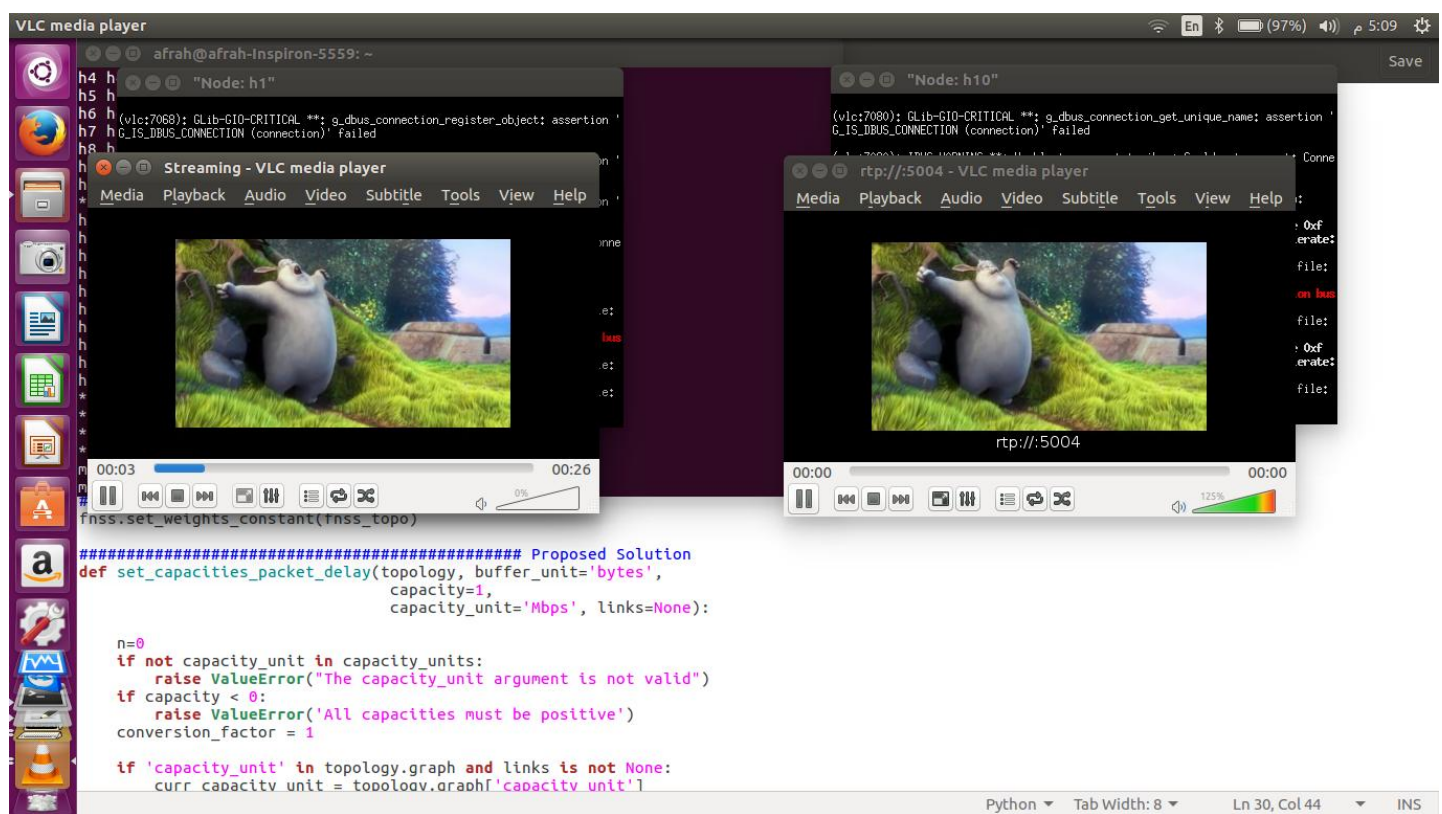

Figure12: Video Performance after Implementing Genetic Algorithm

\section{REFERENCES}

[1] L. Saino, C. Cocora, and G. Pavlou, A Toolchain for Simplifying Network Simulation Setup, in Proceedings of the 6th International ICST Conference on Simulation Tools and Techniques (SIMUTOOLS '13), Cannes, France, March 2013.

[2] T. Ahmed, Resources Management in Software Defined Environment.

[3] H. Li, Resource Optimizations in SoftwareDefined Networking, dissertation submitted in partial fulfillmentof the requirements for degree of doctor of philosophy in computer science and engineering, Ph.D, 2015.

[4] R. Mijumbi, J. Serrat, J. Rubio-Loyolay, N.Boutenz, F. D.Turckz and S.Latr'ex. Dynamic Resource Management in SDNbasedVirtualized Networks, ISBN 978-3-901882-67-8, 10th CNSM and Workshop @2014 IFIP.

[5] D.Tuncer, M. Charalambides, S.Clayman, and G.Pavlou, Adaptive Resource Management and Controlin Software Defined Networks.

[6] T.Zinner, M. Jarschel, A. Blenk, F. Wamser, W. Kellerer, Dynamic Application-Aware Resource ManagementUsing Software-Defined Networking: Implementation Prospects and Challenges, IEEE International Workshop on Quality of Experience Centric Management (QCMan), 2014, 10.1109n/noms.2014.6838404.

[7] S. Kang, G. Kwon, Load Balancing Strategy of SDN Controller Based on Genetic Algorithm, Advanced Science and Technology LettersVol.129 (Mechanical Engineering 2016), pp.219-222.

[8] A. Marotta, Architectures and Algorithms forResource Management in Virtualized Cloud Data Centers, a doctoral thesis in the Department of Electric Engineering and Information TechnologiesMarch 2015.

[9] I.Baran, Adaptive Algorithms for Problems InvolvingBlack-Box Lipschitz Functions, Master thesis at the Massachusetts institute of technology, June 2004.

[10] D. Jakobovic and M. Golub, Adaptive Genetic Algorithm, Journal of Computing and Information Technology - CIT, 1999, 3, 229-235.

[11] R. Maniu and L. A. Dumitru, Exploring the Possibilities of a SelfRegulating SDN Controller, "MirceacelBatran" Naval Academy Scientific Bulletin, Volume XVIII - 2015 - Issue 1.

[12] A.S. Dawood, M.N. Abdullah, A Survey and a Comparative Study on Software-Defined Networking, International Research Journal of Computer Science (IRJCS) ISSN: 2393-9842, issue 08, Volume 3 (August 2016).

[13] http://openvswitch.org/

[14] https://www.sdxcentral.com/sdn/definitions/sdn-controllers/opensource-sdn-controllers/what-is-floodlight-controller/ 